\title{
Perfil clínico e sociodemográfico de adolescentes que permaneceram e não permaneceram no tratamento em um CAPSad de Cuiabá/MT
}

\author{
Clinical and sociodemographic profile of adolescents who stayed \\ and did not stay in treatment at CAPSad Cuiabá/MT
}

Nayara Bueno de Araujo1, Samira Reschetti Marcon², Naiara Gajo Silva², José Roberto Temponi de Oliveira ${ }^{3}$

\section{RESUMO}

Objetivo: Comparar o perfil clínico e sociodemográfico dos adolescentes que permaneceram e que não permaneceram no tratamento no Centro de Atenção Psicossocial para Álcool e outras Drogas (CAPSad). Método: Estudo transversal, baseado no registro de prontuários de 125 usuários que estiveram em tratamento no CAPSad Adolescer em Cuiabá/MT, no período de junho de 2009 a junho de 2011. Resultados: A permanência no tratamento foi predominante no sexo masculino, entre usuários que fizeram uso pesado de substâncias psicoativas, encaminhados ao serviço pelos setores social e judicial, que possuíam relação

\section{Palavras-chave}

Adolescentes, abuso de substâncias psicoativas, pacientes desistentes do tratamento.

\section{Keywords}

Adolescents, drug abuse, patient dropouts. familiar conflituosa e que tinham a participação frequente da família no tratamento. A maior proporção dos que não permaneceram ocorreu na falta de integração do serviço com outros dispositivos da rede. Conclusão: Os achados deste estudo sobre as características dos adolescentes e os fatores intervenientes na permanência reforçam a responsabilidade do CAPS na efetivação da articulação da rede, além de reafirmar que a família deve ser cada vez mais aproximada do tratamento, sendo parte fundamental no projeto de intervenção.

\section{ABSTRACT}

Objective: Compare clinical and sociodemographic profile of adolescents who stayed and did not stay in treatment at Centro de Atenção Psicossocial para Álcool e outras Drogas (CAPSad). Method: A transverse study based on medical records of 125 users who had been in treatment at CAPS ad Adolescer in Cuiabá/MT, from June 2009 to June 2011. Results: The permanence in treatment was predominantly male among users who extensively used psychoactive substances, who were sent for this treatment by social and judicial sectors. They had a conflictual family relationship and also had a frequent participation of family in treatment. The biggest proportion of those who did not stay in treatment happened because of the lack of integration with other devices of the network. Conclusion: To know the characteristics of the adolescents and the interveners factors in the permanence reinforce the responsibility of CAPS in the effective articulation of the network, and moreover, reassure that family must be more and more close to treatment, as an fundamental part in the project of intervention.

1 Universidade Federal de Mato Grosso (UFMT).

2 UFMT, Departamento de Enfermagem.

3 UFMT, Instituto de Ciências Exatas e da Terra, Departamento de Estatística.

Endereço para correspondência: Nayara Bueno de Araujo

Rua Castro Alves, 6, Bairro Santa Cruz

78068-200 - Cuiabá, MT, Brasil

E-mail: naybueno@hotmail.com 


\section{INTRODUÇÃO}

A atenção à saúde mental no Brasil, nos últimos 20 anos, passou por um redirecionamento de modelo de assistência centrado no hospital psiquiátrico para um modelo comunitário, caracterizado pela construção de uma rede de serviços extra-hospitalar articulada a dispositivos de atenção à saúde como os Centros de Atenção Psicossocial (CAPS)'.

O CAPS para usuários de álcool e outras drogas (CAPSad) destaca-se pelo atendimento especializado a pessoas que fazem uso prejudicial dessas substâncias, realizando o acompanhamento clínico, reinserindo o usuário na sociedade e incentivando sua autonomia, além de ter como estratégia de atenção a redução de danos, que visa minimizar os danos individuais e sociais causados pelo uso das substâncias psicoativas (SPA), e considera o usuário como protagonista de seu tratamento'.

Estudos epidemiológicos evidenciam a iniciação dessas substâncias cada vez mais precocemente, o que pode acarretar prejuízos inestimáveis na vida dos adolescentes ${ }^{2}$. De acordo com o V Levantamento Nacional sobre o Consumo de Drogas Psicotrópicas entre Estudantes do Ensino Fundamental e Médio da Rede Pública de Ensino em 17 Capitais Brasileiras, realizado em 2004, a média de idade, em anos, do primeiro uso é de 12,5 para álcool, 12,8 para tabaco, 13,9 para maconha e 14,4 para cocaína ${ }^{3}$.

Um levantamento sobre o uso de álcool, tabaco e outras drogas por adolescentes escolares em município do sul do Brasil identificou uma prevalência da experimentação de drogas entre essa população, especialmente do álcool, e $60,7 \%$ dos adolescentes relataram seu uso pelo menos uma vez na vida e 33\% afirmaram o uso nos últimos 30 dias".

Diante desse quadro e das particularidades da população adolescente que faz uso de SPA, diversas estratégias de intervenção e programas de tratamento têm sido implementados e avaliados ${ }^{5}$, entretanto a literatura aponta que a adesão ao tratamento tem se mostrado um desafio em diferentes contextos, principalmente entre essa população $0^{6,7}$.

Dessa forma, os resultados encontrados em uma pesquisa sobre o perfil sociodemográfico e a adesão ao tratamento de dependentes do álcool em um CAPSad apontam a permanência média menor que seis meses para a faixa etária abaixo de 18 anos $^{8}$, e ainda, em um estudo comparando os perfis de pacientes que aderiram e que não aderiram ao tratamento em um CAPSad, foi observado que o paciente com maior dificuldade em aderir ao tratamento é mais jovem, faz uso de drogas ilícitas, tem menos tempo de uso e, aparentemente, menor estruturação familiar 9 . Essa relação entre a adesão do adolescente ao tratamento para abuso de SPA e seu relacionamento com a família é apontada também na literatura internacional ${ }^{10}$.

Um estudo sobre as relações entre o processo de tratamento de abuso de SPA e os seus resultados aponta que ser mais velho, do sexo masculino e estar envolvido com a justi- ça tem significativa relação com a permanência mais longa no tratamento"1.

Em uma análise da relação entre o estágio motivacional e a adesão ao tratamento de adolescentes usuários de drogas, evidenciou-se que $69,3 \%$ dos adolescentes não aderiram ao tratamento, demonstrando, ainda, que a maioria deles não reconhecia ter problemas em relação ao uso de drogas ${ }^{6}$.

A adesão ao tratamento tem sido amplamente discutida na literatura ${ }^{12,13}$. Uma análise crítica da produção bibliográfica latino-americana no período de 1995 a 2005, acerca da adesão/não adesão ao tratamento de pessoas portadoras de problemas crônicos de saúde, apontou que a ideia recorrente nas definições de adesão/não adesão revela um papel reduzido do paciente no processo de aderir ao tratamento, considerando-o submisso ao profissional e ao serviço de saúde, além de conferir a ele (paciente) maior carga de responsabilidade pela adesão/não adesão ao tratamento, eximindo o profissional da responsabilidade sobre as consequências dessa decisão ${ }^{14}$.

Portanto, considerando que os significados encontrados para a palavra adesão estão fortemente relacionados à obediência do paciente em seguir um tratamento "imposto" pelo profissional de saúde, pressupondo seu papel passivo, e ainda o fato de que a estratégia de redução de danos vai de encontro com essas definições, neste estudo foi utilizado o termo permanência no tratamento, que denota a presença e a continuidade do usuário no serviço, tendo o adolescente a autonomia e os profissionais a corresponsabilidade pelo processo terapêutico.

Diante disso, realizamos este estudo que tem como objetivo comparar o perfil sociodemográfico e clínico dos adolescentes que permaneceram e os que não permaneceram no tratamento no CAPSad em Cuiabá/MT.

\section{MÉTODO}

\section{Desenho e local do estudo}

Estudo transversal, baseado no registro de prontuários, realizado no CAPSad Adolescer, especializado no tratamento de crianças e adolescentes que fazem uso abusivo de álcool e outras drogas. Esse CAPSad funciona com uma equipe multiprofissional de nível médio e superior. São oferecidos atendimentos individuais e/ou grupais, tanto para os usuários como para os familiares, além de oficinas em que ambos participam de acordo com o projeto terapêutico previamente estabelecido.

\section{Amostra}

A amostra do estudo foi composta por 125 prontuários dos usuários do CAPSad Adolescer que estiveram em tratamento no período de junho de 2009 a junho de 2011, com registro de Autorização de Procedimento de Alta Complexidade/ Custo (APAC). 
Critérios de inclusão: prontuários de que constavam ficha de identificação (preenchida pela secretária do CAPS) e ficha de acolhimento e de evolução diária do usuário com as informações necessárias para o estudo (ambas preenchidas por qualquer profissional da equipe).

Critérios de exclusão: prontuários de usuários encaminhados a centros de detenção e/ou instituições para menores que foram a óbito, adolescentes encaminhados ao serviço por ordem judicial, mas que não faziam uso de álcool e outras drogas e que não deram continuidade ao tratamento por estar trabalhando.

\section{Coleta de dados}

Para a coleta de dados, foi construída uma lista com os nomes dos usuários que estiveram no serviço e que geraram APAC no período de estudo. Considerando que alguns usuários tinham mais de um registro de APAC, realizou-se uma conferência manual para verificação dos nomes em duplicidade, gerando uma listagem final com 172 usuários. Desses, sete prontuários não foram encontrados e 40 foram excluídos (11 sem as informações necessárias, 4 encaminhados a centro de detenção, 2 óbitos, 18 não faziam uso de SPA, 5 trabalhando), totalizando 125 prontuários válidos que foram divididos em dois grupos: os que permaneceram no serviço (PS) $n=56$ e os que não permaneceram no serviço (NPS) $n=69$. A coleta dos dados foi realizada em setembro e outubro de 2011.

Para a definição da variável permanência no serviço, considerou-se a frequência do usuário de pelo menos três meses consecutivos em uma das modalidades oferecidas pelo CAPSad, com base nos estudos publicados na literatura brasileira ${ }^{8,9}$.

\section{Instrumento para coleta de dados}

O instrumento para a coleta de dados foi elaborado pelos pesquisadores, sendo composto pelas seguintes variáveis:

Variáveis sociodemográficas: sexo, faixa etária, situação conjugal, nível educacional, ocupação e envolvimento com a justiça;

Variáveis relativas às substâncias psicoativas utilizadas: substâncias psicoativas utilizadas atualmente; frequência do uso: pesado (uso de SPA 20 vezes ou mais ao mês), frequente (uso de SPA seis vezes ou mais ao mês) e no mês (uso de SPA pelo menos uma vez ao mês) ${ }^{15}$ e idade inicial do uso.

Variáveis relacionadas ao tratamento no serviço: tipo de encaminhamento ao serviço: social (serviços ligados à Secretaria de Assistência Social), judicial, familiar e saúde; busca ativa realizada pelo CAPSad; e integração do serviço com outros dispositivos da rede psicossocial. Para essa variável, consideraram-se os casos em que o CAPSad tivesse desenvolvido uma ou mais atividades conjuntas com serviços de saúde, escolas, lazer, comunidade e outros.

Variáveis familiares: tipo de relação familiar; participação familiar no tratamento: pontual (família que participou até três vezes no tratamento), frequente (acima de três participa- ções), nenhuma participação; e presença de familiar responsável pelo tratamento.

Foi realizado um teste piloto do instrumento com 10 prontuários para verificar sua aplicabilidade. Posteriormente, o mesmo foi readequado e aplicado pela pesquisadora principal.

\section{Análise dos dados}

Para análise descritiva, foram apresentadas a frequência simples e a porcentagem. $\mathrm{Na}$ análise inferencial, utilizou-se o teste Qui-quadrado com nível de significância de 5\%. A análise dos dados foi realizada com auxílio do programa estatístico Statistical Package for Social Sciences (SPSS) versão 17.

\section{Considerações éticas}

Este estudo foi precedido da aprovação pelo Comitê de Ética e Pesquisa do Hospital Universitário Júlio Muller, sob o registro no 095/CEP-HUJM/11.

\section{RESULTADOS}

A tabela 1 indica que o perfil sociodemográfico dos adolescentes do CAPSad apresenta proporções semelhantes entre o grupo PS e NPS.

Tabela 1. Comparação das características sociodemográficas entre os adolescentes que permaneceram e os que não permaneceram no CAPSad Cuiabá - MT, junho/2009 a junho/2011

\begin{tabular}{|c|c|c|c|}
\hline Variável & $\begin{array}{c}\mathrm{PS}(\mathrm{n}=56) \\
\mathrm{n}(\%)\end{array}$ & $\begin{array}{c}\mathrm{NPS}(\mathrm{n}=69) \\
n(\%)\end{array}$ & $p$-valor* \\
\hline Sexo & & & 0,026 \\
\hline Masculino & $49(87,5)$ & $49(71,0)$ & \\
\hline Feminino & $7(12,5)$ & $20(29,0)$ & \\
\hline Faixa etária & & & 0,951 \\
\hline $11-12$ anos & $2(3,6)$ & $3(4,3)$ & \\
\hline 13-14 anos & $9(16,0)$ & $10(14,5)$ & \\
\hline $15-17$ anos & $45(80,4)$ & $56(81,2)$ & \\
\hline Situação conjugal** & & & 0,084 \\
\hline Solteiro & $52(98,1)$ & $65(94,2)$ & \\
\hline Casado & $1(1,9)$ & $4(5,8)$ & \\
\hline Nível educacional & & & 0,654 \\
\hline Fundamental incompleto & $48(85,7)$ & $61(88,4)$ & \\
\hline Fundamental completo & $8(14,3)$ & $8(11,6)$ & \\
\hline Ocupação** & & & 0,304 \\
\hline Sim & $7(12,7)$ & $13(19,7)$ & \\
\hline Não & $48(87,3)$ & $53(80,3)$ & \\
\hline Envolvimento com a justiçä** & & & 0,628 \\
\hline Sim & $37(74,0)$ & $46(78,0)$ & \\
\hline Não & $13(26,0)$ & $13(22,0)$ & \\
\hline
\end{tabular}

* Teste Qui-quadrado com significância de 5\%.

** Número de prontuários que não tinham esta informação disponível: Situação conjugal (3); Ocupação (4); Envolvimento justiça (16). 
Em relação ao sexo, a tabela aponta que, entre os que permaneceram no serviço, foi predominante o sexo masculino $(87,5 \%)$ em relação ao sexo feminino $(12,5 \%)$, sendo essa diferença estatisticamente significante $(p=0,026)$.

$\mathrm{Na}$ tabela 2, evidencia-se que a maconha foi a SPA mais utilizada (PS $=84,0 \%$ versus NPS $=74,0 \%$ ), seguida pelo tabaco (PS $=62,5 \%$ versus NPS $=55,0 \%$ ) em ambos os grupos.

Tabela 2. Comparação das variáveis relativas às substâncias psicoativas utilizadas entre os adolescentes que permaneceram e os que não permaneceram no CAPSad Cuiabá - MT, junho/2009 a junho/2011

\begin{tabular}{lccc}
\hline Variável & $\begin{array}{c}\text { PS }(\mathbf{n}=56) \\
\mathbf{n}(\%)\end{array}$ & $\begin{array}{c}\text { NPS }(\mathbf{n}=69) \\
\mathbf{n}(\%)\end{array}$ & p-valor* \\
\hline $\begin{array}{l}\text { Substância psicoativa utilizada } \\
\text { atualmente }\end{array}$ & & & \\
Maconha & $47(84,0)$ & $51(74,0)$ & \\
Tabaco & $35(62,5)$ & $38(55,0)$ & \\
Pasta base & $26(46,4)$ & $33(47,8)$ & \\
Álcool & $25(44,6)$ & $33(47,8)$ & 0,025 \\
Cocaína & $24(42,8)$ & $34(49,3)$ & \\
Frequência do uso*** & & & \\
Pesado & $40(85,1)$ & $36(62,1)$ & \\
Frequente & $4(8,5)$ & $16(27,6)$ & \\
No mês & $3(6,4)$ & $6(10,3)$ & \\
Idade inicial do uso & & $13,17 \pm 1,95$ & \\
(x $\pm D P)$ & $12,62 \pm 1,99$ &
\end{tabular}

* Teste qui-quadrado com significância de $5 \%$.

** Impossibilidade de realização de teste estatístico por se tratar de poliusuários. A amostra não totaliza 125, pois 0 usuário pode ter utilizado mais de uma substância psicoativa.

*** Número de prontuários que não tinham essa informação disponível: 20

Quanto à frequência do uso, a maioria dos usuários pertencia à categoria de uso pesado, com maior proporção no grupo PS (PS $=85,1 \%$ versus NPS $=62,1 \%$ ), sendo essa diferença estatisticamente significante $(p=0,025)$.

A média de idade inicial do uso de SPA foi menor no grupo PS $(12,62 \pm 1,99)$ em relação ao grupo NPS $(13,17 \pm 1,95)$.

A tabela 3 aponta o encaminhamento ao serviço por meio social (PS $=53,6 \%$ versus NPS $=45,6 \%$ ) e judicial (PS = $28,6 \%$ versus NPS $=30,9 \%$ ), com maiores prevalências. Para a busca ativa, o contato telefônico demonstrou maior proporção em ambos os grupos (PS $=58,9 \%$ versus NPS $=63,2 \%$ ).

Em relação à integração do serviço com outros dispositivos da rede, predominou a categoria não integrou $(89,9 \%)$ no grupo NPS, com diferença estatisticamente significante entre os grupos $(p=0,000)$.

Observa-se na tabela 4 que, para o encaminhamento judicial, a categoria uso pesado permaneceu mais no serviço $(85,7 \%)$, com diferença estatisticamente significante $(p=0,020)$ em relação aos que não permaneceram $(64,7 \%)$. O mesmo ocorreu para o encaminhamento social e frequência de uso pesado, com o grupo PS apresentando prevalência de $88,5 \%$, $(p=0,013)$.
Tabela 3. Comparação das variáveis relacionadas ao tratamento no serviço entre adolescentes que permaneceram e os que não permaneceram no CAPSad Cuiabá - MT, junho/2009 a junho/2011

\begin{tabular}{lccc}
\hline Variável & $\begin{array}{c}\text { PS }(\mathrm{n}=56) \\
\mathbf{n}(\%)\end{array}$ & $\begin{array}{c}\mathrm{NPS}(\mathrm{n}=69) \\
\mathbf{n}(\%)\end{array}$ & p-valor* \\
\hline $\begin{array}{l}\text { Encaminhamento ao serviç*** } \\
\text { Social }\end{array}$ & $30(53,6)$ & $31(45,6)$ & 0,615 \\
Judicial & $16(28,6)$ & $21(30,9)$ & \\
Família & $5(8,9)$ & $11(16,1)$ & \\
Saúde & $5(8,9)$ & $5(7,4)$ & \\
Busca ativa** & & & 0,093 \\
Contato telefônico & $33(58,9)$ & $43(63,2)$ & \\
Visita domiciliar & $22(39,3)$ & $17(25,1)$ & \\
Contato telefônico e visita domiciliar & $0(0,0)$ & $2(2,9)$ & \\
Nenhum tipo de busca & $1(1,8)$ & $6(8,8)$ & \\
Integração do serviço com outros dispositivos & & & 0,000 \\
da rede & & & \\
Sim & $29(51,8)$ & $7(10,1)$ & \\
Não & $27(48,2)$ & $62(89,9)$ & \\
\hline
\end{tabular}

* Teste qui-quadrado com significância de $5 \%$.

** Número de prontuários que não tinham essa informação disponível: encaminhamento ao serviço (1); busca ativa (1).

Tabela 4. Relação entre tipo de encaminhamento ao serviço, frequência de uso de substâncias psicoativas e permanência e não permanência dos adolescentes no CAPSad Cuiabá - MT, junho/2009 a junho/2011

\begin{tabular}{lcccc}
\hline $\begin{array}{l}\text { Encaminhamento } \\
\text { ao serviço** }\end{array}$ & $\begin{array}{c}\text { Frequência do } \\
\text { uso** }\end{array}$ & $\begin{array}{c}\text { PS }(\mathbf{n}=56) \\
\mathbf{n}(\%)\end{array}$ & $\begin{array}{c}\text { NPS }(\mathbf{n}=69) \\
\mathbf{n}(\%)\end{array}$ & p-valor* \\
\hline Judicial & Pesado & $12(85,7)$ & $11(64,7)$ & 0,020 \\
& Frequente & $0(0)$ & $6(35,3)$ & \\
& No mês & $2(14,3)$ & $0(0)$ & \\
Social & Pesado & $23(88,5)$ & $15(55,6)$ & 0,013 \\
& Frequente & $3(11,5)$ & $6(22,2)$ & \\
& No mês & $0(0)$ & $6(22,2)$ & \\
Saúde & Pesado & $2(66,7)$ & $4(100,0)$ & 0,212 \\
& Frequente & $1(33,3)$ & $0(0)$ & \\
& No mês & $0(0)$ & $0(0)$ & \\
Família & Pesado & $3(75,0)$ & $5(55,6)$ & 0,123 \\
& Frequente & $0(0)$ & $4(44,4)$ & \\
& No mês & $1(25,0)$ & $0(0)$ & \\
\hline
\end{tabular}

* Teste qui-quadrado com significância de $5 \%$.

** Número de prontuários que não tinham essa informação disponível: encaminhamento ao serviço (1); frequência do uso (20).

A tabela 5 evidencia na variável relação familiar a categoria relação conflituosa em maior prevalência no grupo PS (59,6\%), enquanto no grupo NPS predominou a relação satisfatória $(54,7 \%)$, com diferença estatisticamente significante entre os grupos $(p=0,030)$. 
Tabela 5. Comparação das variáveis relacionadas à família entre os adolescentes que permaneceram e os que não permaneceram no CAPSad Cuiabá - MT, junho/2009 a junho/2011

\begin{tabular}{lccc}
\hline Variável & $\begin{array}{c}\text { PS }(\mathrm{n}=56) \\
\mathrm{n}(\%)\end{array}$ & $\begin{array}{c}\mathrm{NPS}(\mathrm{n}=69) \\
\mathrm{n}(\%)\end{array}$ & p-valor* \\
\hline $\begin{array}{l}\text { Relaçãofamiliar** } \\
\text { Conflituosa }\end{array}$ & $31(59,6)$ & $28(43,7)$ & 0,030 \\
$\begin{array}{l}\text { Satisfatória } \\
\text { Inexistente }\end{array}$ & $17(32,7)$ & $35(54,7)$ & \\
Participação familiar no tratamento & $4(7,7)$ & $1(1,6)$ & \\
$\begin{array}{l}\text { Frequente } \\
\text { Pontual }\end{array}$ & $27(48,2)$ & $18(26,1)$ & \\
$\quad$ Nenhuma & $13(23,2)$ & $37(53,6)$ & \\
Familiar responsável pelo tratamento** & $16(28,6)$ & $14(20,3)$ & \\
Sim & & & 0,361 \\
Não & $40(72,7)$ & $55(79,7)$ & \\
\hline
\end{tabular}

* Teste qui-quadrado com significância de 5\%.

** Número de prontuários que não tinham essa informação disponível: relação familiar (9); familiar responsável pelo tratamento (1).

Quanto à variável participação familiar no tratamento, o grupo PS teve maior prevalência para a participação frequente $(48,2 \%)$, enquanto no grupo NPS essa participação foi pontual $(53,6 \%)$, sendo essa diferença entre os grupos estatisticamente significante $(p=0,002)$. A tabela aponta, ainda, que a maioria dos usuários tinha um familiar responsável pelo tratamento em ambos os grupos PS e NPS (72,7\% e $79,7 \%$, respectivamente).

\section{DISCUSSÃO}

A análise do perfil sociodemográfico da população estudada aponta que, em ambos os grupos, houve predomínio de usuários do sexo masculino, de 15 a 17 anos, solteiro, com ensino fundamental incompleto, sem ocupação e que já tiveram envolvimento com a justiça. Os dados evidenciados em estudo com adolescentes usuários de substâncias ilícitas em Porto Alegre/RS corroboram esses achados ao evidenciar a prevalência do sexo masculino em 96,1\% da amostra, com média de idade de 16,3 anos e ensino fundamental incompleto $(79,6 \%)^{6}$.

Um estudo de caracterização dos adolescentes internados por uso de álcool e outras drogas em Curitiba/PR também identificou entre a população a predominância do sexo masculino (85,8\%), no entanto a maioria dos adolescentes estava fora da escola (62,4\%), não possuía nenhum tipo de ocupação, além de ter relatado alta incidência $(63,7 \%)$ de infrações e envolvimentos com a polícia ${ }^{16}$.

O II levantamento domiciliar sobre o uso de drogas psicotrópicas no Brasil evidenciou, na região Centro-Oeste, o envolvimento precoce dos entrevistados de 12 a 17 anos com as drogas e a dependência, e em relação à escolaridade, 51,7\% dessa população não eram letrados ou possuíam apenas 0 ensino fundamental incompleto ${ }^{17}$. Portanto, as características dos adolescentes usuários de álcool e outras drogas do CAPSad assemelham-se às da população adolescente em geral da região Centro-Oeste, bem como às dos estudos descritos.

A comparação da variável sexo entre os usuários do grupo PS e NPS demonstrou a predominância dos homens entre os que permaneceram em relação às mulheres. Esse achado corrobora os dados obtidos em estudo realizado em um CAPSad de Campo Grande/MS, que aponta que apenas 10,2\% das mulheres aderiram ao tratamento 9 . O abandono do tratamento de mulheres usuárias de álcool em um ambulatório especializado em dependência química em São Paulo/SP foi de $53,1 \%$ no primeiro mês de tratamento, $13,0 \%$ no segundo e $6,8 \%$ no terceiro ${ }^{18}$.

A baixa prevalência do sexo feminino no tratamento de álcool e outras drogas pode ser justificada pelo preconceito que atinge a mulher que faz uso abusivo dessas substâncias, além de sentimentos de culpa, vergonha, medo e baixa autoestima, que as levam a evitar que alguém descubra seu problema ${ }^{19,20}$. Cabe ressaltar que, em estudo sobre o alcoolismo feminino realizado em uma Unidade de Tratamento de Alcoolistas no Rio de Janeiro/RJ, foi observado que 70,0\% das usuárias que faziam parte do Grupo de Mulheres aderiram ao tratamento, em contrapartida 90,0\% evadiram do serviço antes da criação desse grupo ${ }^{21}$.

Em relação às variáveis relativas às SPA utilizadas, identificou-se que a maconha teve maior prevalência de uso nos dois grupos. Resultados semelhantes foram obtidos com adolescentes internados por dependência de drogas ${ }^{6}$. Confrontando com esses achados, estudos apontam que a substância mais utilizada por adolescentes é o álcool3,4, entretanto deve-se considerar que nesses estudos foram investigados adolescentes escolares da população em geral, e não apenas os que faziam uso abusivo de álcool e outras drogas.

Quanto à frequência do uso de SPA, os usuários que se enquadraram na categoria de uso pesado permaneceram mais no serviço do que os que faziam uso frequente ou no mês, e, ao relacionar esse achado com a variável encaminhamento ao serviço, obteve-se que o adolescente que faz uso pesado de SPA e que foi encaminhado ao serviço por ordem judicial ou social permaneceu mais no serviço em comparação com os outros grupos. Esse fato sugere que o uso pesado de álcool e outras drogas pode gerar graves consequências sociais, e uma possível hipótese para que esse adolescente tenha permanecido mais no serviço pode estar relacionada com a obrigatoriedade do tratamento na ocasião do encaminhamento.

A obrigatoriedade do tratamento tem se mostrado controversa, e, por um lado, autores apontam que pessoas sub- 
metidas a tratamentos coercitivos podem sofrer diferentes tipos de pressões externas para mudar um comportamento-problema, e a coação legal pode ampliar um compromisso pessoal para diminuir o uso de drogas, alcançando resultados relevantes ${ }^{6}$. Em contraposição, os encaminhamentos judiciais, atualmente denominados como "justiça terapêutica", impõem ao usuário forçosamente uma mudança de comportamento para torná-lo uma pessoa socialmente adequada, ou seja, que não usa SPA. Presume-se que a ideia de abstinência reflita um valor compartilhado por todos, e pretende-se impor tal conduta àqueles que transgridam a proibição ${ }^{22}$. No entanto, essa proposta se opõe à estratégia de redução de danos e se integra a um modelo tradicional e proibicionista. Assim, esses achados apontam a necessidade de que estudos específicos discutindo essa temática, ainda tão polêmica na sociedade, sejam realizados.

Ao analisar a idade inicial do uso de SPA, foi evidenciada sua precocidade, com média de 12,62 anos no grupo de adolescentes que permaneceram no serviço e 13,17 anos no grupo que não permaneceu. Estudo aponta que 66,0\% dos adolescentes internados por uso de álcool e outras drogas iniciaram sua experiência com SPA entre os 12 e 15 anos, embora conste também a primeira experimentação entre 6 e 10 anos de idade ${ }^{16}$. Outro estudo que corrobora esses dados identificou idade média de experimentação de drogas (exceto álcool e tabaco) de 13,0 anos por adolescentes escolares".

A relação entre a idade e o resultado do tratamento aponta que quanto menor a idade do usuário, menores as chances de permanência no tratamento ${ }^{23} \mathrm{e}$, ainda, quanto mais precoce a idade de início de SPA, maior o risco de desenvolvimento de dependência ${ }^{24}$, e os prejuízos provocados pelas drogas podem ir desde uma intoxicação ou overdose até alterações mais duradouras ou irreversíveis².

Em relação ao tipo de encaminhamento ao serviço, o predomínio dos encaminhamentos social e judicial em ambos os grupos pode ser justificado pela associação do uso de álcool e outras drogas com a criminalidade, violência e práticas antissociais', além da presença histórica do setor judicial na condução das demandas desse tipo de população. Cabe ainda destacar o baixo índice de encaminhamentos feitos pelo setor da saúde, o que sugere a falta de articulação entre a rede de serviços, além da necessidade do profissional de saúde de incorporar a atenção ao usuário de álcool e outras drogas em sua prática profissional.

Embora os enfermeiros, em sua prática assistencial, identifiquem as necessidades de saúde entre os usuários de SPA, poucas ações são realizadas devido à falta de capacitação profissional, de implantação local de programas de atenção a esses usuários e à ausência de uma rede social e de serviços que dê sustentação e apoio às ações em saúde ${ }^{25}$.

Quanto ao tipo de busca ativa realizada pelo serviço, o contato telefônico e a visita domiciliar foram as estratégias mais utilizadas. Vale ressaltar que a busca ativa é uma das ações que devem ser priorizadas no CAPSad, considerando a baixa assiduidade e o alto índice de abandono característicos dessa população ${ }^{7}$. A visita domiciliar, embora esteja muitas vezes limitada pela violência que demarca o contexto social em que vive o usuário, contribui na organização do programa de atenção a essa população ${ }^{25}$.

Os resultados demonstraram que os usuários que não tiveram durante o tratamento algum tipo de integração entre os diferentes dispositivos da rede permaneceram menos no serviço em relação aos que tiveram essa integração. Uma possibilidade para esse achado refere-se ao fato de que com a integração do CAPSad-rede efetivada, embora ainda muito discreta como evidenciado neste estudo, o adolescente se reconhece como parte da comunidade/sociedade, o que pode ser um estímulo para a sua permanência no tratamento. Não foram encontrados estudos que pudessem comparar esse achado, no entanto eles reafirmam a importância da integração da rede de atenção à saúde mental, que se estrutura na medida em que são permanentemente articuladas outras instituições, associações, cooperativas e variados espaços das cidades, buscando, assim, garantir a resolutividade e a promoção da autonomia e da cidadania dos usuários?.

Conhecer a estrutura do serviço e as necessidades atuais e/ou potenciais dos usuários orienta, refina e otimiza a proposta terapêutica em andamento ${ }^{26}$.

Em relação às variáveis relacionadas à família, observouse que, entre os adolescentes que permaneceram no tratamento, a relação com a família era conflituosa. Dados semeIhantes foram encontrados em estudo com adolescentes de 12 a 17 anos, referenciados ao tratamento para abuso de drogas, e seus pais, em que a adesão ao tratamento esteve relacionada com a percepção por parte dos adolescentes de intenso conflito familiar, e os adolescentes que se engajaram no tratamento perceberam mais conflito em suas famílias do que os desistentes ${ }^{10}$.

É possível inferir que esse achado tenha ocorrido pelo fato de que o adolescente com dificuldade de relacionamento com a família encontra no serviço um local de amparo e acolhimento. O CAPS, por meio de sua estrutura física e proposta de tratamento, deve exercer a função de apoiar, acolher e promover a saúde, favorecendo a criação de novos vínculos sociais, que havia sido perdido pelo estigma de usuário de drogas ${ }^{27}$.

Quanto à participação familiar no tratamento, observa-se que, no grupo de adolescentes que permaneceram no serviço, a família teve participação frequente no tratamento, enquanto, no grupo que não permaneceu, a família participou de forma pontual. A participação da família no processo de tratamento é fundamental na adesão de adolescentes usuários de drogas ao tratamento, uma vez que a adesão desses pacientes se mostrou dependente do grau de envolvimento e participação da família no processo terapêutico ${ }^{28}$. $O$ apoio 
da família é um fator que auxilia o adolescente a se manter no tratamento ou reduzir o consumo da SPA?

A família coloca-se como protagonista tanto para o desenvolvimento do uso abusivo de drogas quanto para a prevenção desse uso, apontando, dessa forma, a necessidade de tratar o sistema familiar para transformação da dependência em produção de saúde ${ }^{29}$.

\section{Limitações}

No presente estudo, foram encontradas algumas dificuldades relacionadas à busca de informações, pelo fato de essas serem obtidas por meio de dados secundários, o que gerou perdas nos registros dos prontuários, uma vez que esses dados dependiam de quem registrava as informações.

Estudos comparativos com adolescentes usuários de SPA são escassos no Brasil e especificamente no estado de Mato Grosso, assim apresentamos como limitação metodológica o número reduzido de estudos com a temática, dificultando a comparação dos dados com a realidade brasileira.

\section{CONCLUSÕES}

Na comparação do perfil sociodemográfico e clínico dos adolescentes que permaneceram e dos que não permaneceram no CAPSad, evidenciamos um baixo índice de permanência dessa população no serviço e uma semelhança nos perfis sociodemográfico e clínico entre os grupos PS e NPS. Identificaram-se as variáveis: sexo masculino, frequência do uso pesado de SPA, integração do serviço com outros dispositivos da rede, tipo de relação familiar conflituosa e a participação frequente da família no tratamento, como fatores que se associaram à permanência dos adolescentes no CAPSad.

Conhecer os fatores intervenientes na permanência reforça a responsabilidade do CAPS na efetivação da articulação da rede com os mais diferenciados dispositivos, além de reafirmar que a família deve ser cada vez mais aproximada do tratamento, sendo parte fundamental no projeto de intervenção.

Ressaltamos a necessidade de que novos estudos sejam realizados a fim de melhor esclarecimento sobre essa população, visando ampliar o olhar e, consequentemente, as intervenções focadas nas especificidades e necessidades individuais, contribuindo para a melhoria da qualidade de vida desses adolescentes.

\section{REFERÊNCIAS}

1. Brasil. Ministério da Saúde. Secretaria de Atenção à Saúde. Departamento de Ações Programáticas Estratégicas. Coordenação Geral de Saúde Mental. Reforma Psiquiátrica e Política de Saúde Mental no Brasil. Documento apresentado à Conferência Regional de Reforma dos Serviços de Saúde Mental: 15 anos depois de Caracas. OPAS. Brasília; 2005.
2. Marques ACPR, Cruz MS. 0 adolescente e 0 uso de drogas. Rev Bras Psiquiatr. 2000;22(Supl 2):S32-6.

3. Galduróz JCF, Noto AR, Fonseca AM, Carlini EA. V Levantamento Nacional sobre o Consumo de Drogas Psicotrópicas entre Estudantes do Ensino Fundamental e Médio da Rede Pública de Ensino nas 27 Capitais Brasileiras - 2004. São Paulo: CEBRID; 2005.

4. Vieira PC, Aerts DRGC, Freddo SL, Bittencourt A, Monteiro L. Uso de álcool, tabaco e outras drogas por adolescentes escolares em município do sul do Brasil. Cad Saude Publica. 2008;24(11).

5. Almeida MM, Oliveira MA, Pinho PH. 0 tratamento de adolescentes usuários de álcool e outras drogas: uma questão a ser debatida com os adolescentes? Rev Psiq Clín. 2008;35(Supl 1):S76-81.

6. Oliveira MS, Szupszynski K, Del R, Diclemente C. Estudo dos estágios motivacionais no tratamento de adolescentes usuários de substâncias psicoativas ilícitas. Psico. 2010;41(1):40-6

7. Vasters GP, Pillon SC. 0 uso de drogas por adolescentes e suas percepçoes sobre adesão e abandono de tratamento especializado. Rev Latino-Am Enfermagem. 2011;19(2):317-24.

8. Monteiro CFS, Fé LCM, Moreira MAC, Albuquerque IEM, Silva MG, Passamani MC. Perfil sociodemográico e adesão ao tratamento de dependentes de álcool em CAPSad do Piauí. Esc Anna Nery Rev Enferm. 2011;15(1):90-5.

9. Peixoto C, Prado CHO, Rodrigues CP, Cheda JND, Mota LBT, Veras AB. Impacto do perfil clínico e sociodemográfico na adesão ao tratamento de pacientes de um Centro de Atenção Psicossocial a Usuários de Álcool e Drogas (CAPSad). J Bras Psiquiatr. 2010;59(4):317-21.

10. Dakof GA, Tejeda M, Liddle HA. Predictors of engagement in adolescent drug abuse treatment. J Am Acad Child Adolesc Psychiatry. 2001;40(3):274-81.

11. Hser Y, Evans E, Huang D, Anglin DM. Relationship between drug treatment services, retention, and outcomes. Psychiatr Serv. 2004;55(7):767-74.

12. Gusmão JL, Mion Junior D. Adesão do tratamento - conceitos. Rev Bras Hipertens. 2006;13(1):23-5.

13. Leite SN, Vasconcellos MPC. Adesão à terapêutica medicamentosa: elementos para a discussão de conceitos e pressupostos adotados na literatura. Ciênc Saúde Coletiva. 2003;8(3):775-82.

14. Reiners AAO, Azevedo RCS, Vieira MA, Arruda ALG. Produção bibliográfica sobre adesão/ não adesão de pessoas ao tratamento de saúde. Ciênc Saúde Coletiva. 2008;13(Supl 2):S2299-306

15. Edwards $G$, Arif A. Nomenclature and classification of drug and alcohol-related problems: a WHO, Memorandum. Bull World Health Organ. 1981;59(2):225-45.

16. Alves R, Kossobudzky LA. Caracterização dos adolescentes internados por álcool e outras drogas na cidade de Curitiba. Interação em Psicologia. 2002;6(1):65-79.

17. Carlini EA, Galduróz JC, Noto AR, Carlini CM, Oliveira LG, Nappo SA, et al. Il levantamento domiciliar sobre 0 uso de drogas psicotrópicas no Brasil: estudo envolvendo as 108 maiores cidades do país - 2005. São Paulo: Páginas \& Letras; 2007; p. 472.

18. Elbreder MF, Laranjeira R, Siqueira MM, Barbosa DA. Perfil de mulheres usuárias de álcool em ambulatório especializado em dependência química. J Bras Psiquiatr. 2008;57(1):9-15.

19. Assis DFF, Castro NT. Alcoolismo feminino: início do beber alcoólico e busca por tratamento. Textos \& Contextos. 2010;9(2):358-70.

20. Rossetti ACK, Santos VEP. Dependente químico: adesão ao tratamento. Rev Téc-Cient Enferm. 2006;4(14):34-41.

21. Cesar BAL. Alcoolismo feminino: um estudo de suas peculiaridades - Resultados preliminares. J Bras Psiquiatr. 2006:55(3):208-11.

22. Boiteux L, Ribeiro MM. Justiça terapêutica: redução de danos ou proibicionismo dissimuIado? In: Seibel SD. Dependência de drogas. São Paulo: Atheneu; 2010. p. 1113-22.

23. Beynon CM, McMinn AM, Marr AJ. Factors predicting drop out from, and retention in, specialist drug treatment services: A case control study in the North West of England. BMC Public Health. 2008;8:149.

24. Botelho BHF, Medeiros E. A criança, 0 adolescente e a droga. In: Seibel SD. Dependência de drogas. São Paulo: Atheneu; 2010. p. 977-87.

25. Gonçalves SSPM, Tavares CMM. Atuação do enfermeiro na atenção ao usuário de álcool e outras drogas nos serviços extra-hospitalares. Esc Anna Nery Rev Enferm. 2007:11(4):586-92 
26. Ribeiro M. Organização de serviços para o tratamento da dependência do álcool. Rev Bras Psiquiatr. 2004;26(Supl 1):S59-62.

27. Souza J, Kantorski LP, Mielke FB. Vínculos e redes sociais de indivíduos dependentes de substâncias psicoativas sob tratamento em CAPS AD. SMAD. Rev Eletrônica Saúde Mental Álcool Drog. 2006;2(1).
28. Scivoletto S, Henriques Junior SG, Andrade AG. Proposta de tratamento para adolescentes usuários de drogas - a internação domiciliar como alternativa. Infanto - Rev Neuropsiq da Inf e Adol. 1996;4(1):33-40.

29. Schenker M, Minayo MCS. A importância da família no tratamento do uso abusivo de drogas: uma revisão da literatura. Cad Saude Publica. 2004;20(3):649-59. 